\title{
EXPANSÃO DA CULTURA DO EUCALIPTO NOS MUNICÍPIOS MINEIROS E GESTÃO TERRITORIAL
}

\author{
João Batista Rezende ${ }^{1}$, José Roberto Pereira² ${ }^{2}$ Douglas de Oliveira Botelho ${ }^{3}$
}

(recebido: 16 de setembro de 2011; aceito: 28 de setembro de 2012)

\begin{abstract}
RESUMO: Neste trabalho, trata-se da elaboração do índice de monocultura (IM) do eucalipto como elemento chave para a gestão do território, na perspectiva do desenvolvimento sustentável. Assim, por meio deste, analisa-se a "geografia" do plantio de eucalipto em Minas Gerais, de tal forma que possa subsidiar o planejamento, a organização, o controle e o uso do território. O Índice de Monocultura proposto neste trabalho foi elaborado e verificou-se, pela sua aplicação aos municípios de Minas Gerais, que constitui um instrumento relevante de gestão do território. Verificou-se, ainda, que a cultura do eucalipto representa um baixo índice de monocultura no estado de Minas Gerais, mostrou-se a geografia dessa cultura e suas possibilidades de expansão territorial de forma sustentável. Portanto, conclui-se que os resultados apresentados contribuem para a definição de políticas públicas de gestão do território em Minas Gerais, com possibilidades de aplicar a metodologia em outros estados da federação.
\end{abstract}

Palavras-chave: Monocultura, desenvolvimento sustentável, gestão do território.

\section{EXPANSION OF EUCALYPTUS CULTURE IN THE MUNICIPALITIES OF MINAS GERAIS AND TERRITORIAL MANAGEMENT}

\begin{abstract}
This article deals with the development of monoculture index (MI) of eucalyptus as a key element for the management of the territory, from the perspective of sustainable development. We analyzed the "geography" of the eucalyptus plantation in Minas Gerais, to support the planning, organization, control and use of territory. The Monoculture index proposed, which is an important tool for land management, was developed and validate by its application to municipalities of Minas Gerais state. It was shown that the culture of Eucalyptus represents a low rate of monoculture in the state of Minas Gerais and that the geography of this culture is compatible with sustainable territorial expansion. Therefore, these results contribute to the definition of public land management in Minas Gerais and the methodology used can be applied to other states.
\end{abstract}

Key words: Monoculture, sustainable development, land management.

\section{INTRODUÇÃO}

O presente trabalho é resultado de um estudo realizado no âmbito do projeto Zoneamento EcológicoEconômico do estado de Minas Gerais-ZEE-MG (SCOLFORO et al., 2008). Partiu-se do pressuposto de que se discute muito sobre os impactos econômicos, sociais e ambientais do plantio do eucalipto, tanto no âmbito acadêmico-científico quanto no âmbito empresarial e dos movimentos sociais, mas sem uma base de informações confiável que possa sustentar inferências, análises e conclusões.

Assim, levantam-se algumas questões norteadoras: em que situações se pode considerar o eucalipto uma monocultura, levando em conta a área do território municipal e seu uso atual? Em quais municípios se concentra o plantio dessa cultura? Qual a área possível de expansão dessa cultura em cada município do estado, na perspectiva da ocupação das áreas disponíveis?

No período 2006/2010 observou-se expressivo crescimento das áreas de florestas plantadas no Brasil, em especial de eucalipto em decorrência de seu rápido crescimento, produtividade, vigor e a adaptação a diferentes habitats. Aárea de eucalipto cresceu 1,29 milhão, o equivalente ao crescimento médio anual de $6,6 \%$, enquanto a de pinus vem decrescendo e sendo substituída pelo eucalipto. Em 2010, a área plantada com eucalipto no país atingiu 4,7 milhões de hectares, representando 72,3\% do total das florestas plantadas (ASSOCIAÇÃO BRASILEIRA DE PRODUTORES DE FLORESTAS PLANTADAS - ABRAF, 2011).

Em Minas Gerais, as áreas plantadas representavam $30 \%$ e os maiores agentes reflorestadores nos últimos cinco anos (2006-2010) foram, pela ordem de importância, as

${ }^{1}$ Graduado em Ciências Econômicas, Professor Doutor em Administração - Centro de Estudos em Políticas Públicas Fundação João Pinheiro - Alameda das Acácias, 70, Pampulha - 31275-150 - Belo Horizonte, MG, Brasil - joaobatistarezende@yahoo.com.br ${ }^{2}$ Graduado em Administração Rural, Professor Doutor em Sociologia - Universidade Federal de Lavras/UFLA - Diretoria de Contratos e Convênios/DICON - Prédio da Reitoria - Cx. P. 3037 - 37.200-000 - Lavras, MG, Brasil - jrobpereira25@yahoo.com.br ${ }^{3}$ Administrador, Doutorando em Administração - Universidade Federal de Lavras/UFLA - Programa de Pós-Graduação em Administração Cx. P. 3037 - 37.200-000 - Lavras, MG, Brasil - douglasbotelho@gmail.com 
indústrias siderúrgicas integradas; as independentes (guseiras), as produtoras de celulose (inclusive aquelas com unidades industriais localizadas em outros estados como ES, BA e SP), as produtoras de ferroligas e os produtores independentes. Os quatro primeiros agentes reflorestadores são, também, os maiores consumidores da matéria-prima florestal.

A aprovação, em 2009, da Lei Estadual 18.365 (MINAS GERAIS, 2009), determinou a redução do consumo de carvão de vegetação de origem nativa até o limite máximo de $5 \%$ do consumo anual total a partir de $2019^{4}$ e já provocou reações nos setores consumidores de carvão vegetal, especialmente, entre os grandes consumidores. De acordo com estimativas da Associação Mineira de Silvicultura AMS (2011), o setor reduziu o consumo de carvão de origem nativa, passando de 41,0\% em 2005 para 23,7\% em 2010, e ampliou os plantios florestais. Em 2010, só os setores independentes, guseiro e o de ferroligas, retomaram os plantios, reduzidos em 2008, em decorrência da crise econômica mundial. Foram plantados cerca de $50 \mathrm{mil}$ hectares (38,5\% do total médio anual do estado), superando as estimativas necessárias para suprir $95 \%$ da demanda a partir de 2018.

Quanto à importância econômica, o carvão vegetal é o segmento que mais se destaca no Sistema Agroindustrial Florestal (SAG), em seguida, tem-se a madeira para celulose e outros usos como fonte de energia na indústria, comércio e residências. Embora o estado detenha a maior área em florestas plantadas do país, cerca de $27,2 \%$ do carvão consumido ainda é produzido com madeira extraída de matas nativas, inclusive de outros estados, provocando fortes impactos ambientais negativos. Daí a importância e necessidade de ampliação dos plantios para o atendimento da demanda crescente e redução da pressão sobre os remanescentes nativos. No entanto, o crescimento da área plantada de eucalipto pode caracterizar monocultura.

O termo monocultura, plantio extensivo de um único vegetal é, normalmente, disseminado como algo "negativo" por ser considerado como insustentável. Todavia, a monocultura envolve um balanço entre vantagens e desvantagens. Dentre as desvantagens, segundo Spedding (1997) destacam-se: vulnerabilidade

\footnotetext{
${ }^{4}$ No artigo 47 a Lei Estadual 18.365/2009 determina uma redução gradual do consumo de matéria-prima nativa estabelecendo o limite máximo de $15 \%$ entre 2009 e 2013, 10\% entre 2014 e 2017 e $5 \%$ a partir de 2018 .
}

de todo o empreendimento, simultaneamente às mudanças adversas no clima e a excessiva e crescente dependência dos insumos e matérias-primas. Para Navarro et al. (2005), a monocultura traz desvantagens ambientais, porque exaure o solo e reduz a biodiversidade. As desvantagens sociais ocorrem porque reduz o uso da mão de obra no campo e afugenta as populações rurais. Em termos econômicos pode trazer desvantagens, pois apresenta riscos em relação a doenças, pragas ou queda do preço do produto no mercado, o que pode colocar em risco de perda de toda a cadeia produtiva regional. Os autores ainda destacam várias externalidades negativas da monocultura, como a destruição de florestas nativas, redução das fontes de água superficiais e subterrâneas, problemas de saúde em comunidades vizinha, contaminação das águas e degradação dos solos, situações nem sempre comprovadas.

Entre as vantagens da monocultura, Spedding (1997) cita o alinhamento das necessidades do sistema de produção com os atributos naturais da área; economias de escala, as mesmas habilidades, equipamentos e infraestrutura são aplicados em larga escala, reduzindo os custos unitários de produção; volume e continuidade de suprimento os quais permitem a realização de arranjos de mercado de longo prazo, levando a uma regularidade na oferta dos produtos florestais; maquinário de fácil operação para plantio, tratamento, colheita, produtividade e lucratividade elevadas. A essas vantagens pode-se acrescentar o fato de que o eucalipto plantado em áreas já degradadas pode contribuir para a recuperação das mesmas e reduzir, de maneira significativa, o desmatamento das áreas nativas. Nesse sentido, acredita-se que o eucalipto manejado adequadamente pode contribuir para o desenvolvimento sustentável, ou seja, “[...] quando se consegue, em sua concepção e implementação, um equilíbrio entre crescimento econômico sustentado, melhor distribuição da renda e da riqueza, e qualidade adequada do meio ambiente" (HADDAD, 2004, p. 7).

Portanto, conduziu-se este trabalho, com o objetivo de elaborar um índice de monocultura (IM) do eucalipto e, com base nele, analisar a "geografia" dessa cultura no estado de Minas Gerais, de tal forma que possa subsidiar o planejamento, a organização, o controle e o uso do território estadual e municipal. A relevância dos resultados deste trabalho é poder contribuir para a definição de políticas públicas mais adequadas à realidade do estado e para a efetividade da gestão do território pelos governos estaduais e municipais.

Cerne, Lavras, v. 19, n. 1, p. 1-7, jan./mar. 2013 


\section{MATERIAL E MÉTODOS}

Este trabalho tem como base metodológica a sistematização de dados secundários levantados a partir do Zoneamento Econômico-Ecológico (ZEE) do estado de Minas Gerais como instrumento de desenvolvimento territorial, do Mapeamento e Inventário da Flora Nativa e dos Reflorestamentos de Minas Gerais, bem como das atualizações recentes dos anuários da Associação Mineira de Silvicultura (AMS) e da Associação Brasileira de Produtores de Florestas Plantadas (ABRAF), complementados com informações do Censo Agropecuário 2006, do Instituto Brasileiro de Geografia e Estatística IBGE (2008). A base de informações utilizada é municipal e não foram feitos levantamentos primários de informações.

Para a elaboração do Índice de Monocultura (IM), levou-se em consideração que a monocultura representa o grau de especialização das culturas, ou seja, a proporção da área disponível do município destinada à produção dessas culturas, nesse caso, o eucalipto. Para tal, tomouse como referência o trabalho desenvolvido por Kageyama (2004), a respeito do desenvolvimento rural em municípios paulistas. A autora considerou como monocultura a utilização de $40 \%$ ou mais de área do estabelecimento ocupada com determinada cultura.

Na construção do IM proposto, procurou-se utilizar outras variáveis disponíveis, no sentido de identificar as áreas disponíveis para o plantio de eucalipto, subtraindose da área total do município as áreas inundadas, as áreas ocupadas por unidades de proteção permanente e reserva legal e, ainda, aquelas áreas que poderiam ser ocupadas pela urbanização, estradas, cursos d'água e outras onde não é possível ou desejável o plantio do eucalipto.

Para este estudo, selecionaram-se dez municípios mineiros, separados em dois grupos. No primeiro, foram escolhidos aqueles cinco com a maior área plantada de eucalipto, em 2007. No segundo, foram selecionados aqueles onde foi maior a participação da área de eucalipto, em relação à área total do município.

A fórmula utilizada para o cálculo do índice de monocultura (IM) foi a seguinte: $\operatorname{IM}(\%)=\{$ área cultivada de eucalipto, em ha\} / \{área total do município, em ha[ $\Sigma$ das areas,em ha (inundadas; das unidades de conservação e proteção integral; $20 \%$ da área do município, destinada às reservas legais; $10 \%$ da área do município relativo às áreas urbanas e outros usos)] $* 100\}$

Assim, o índice de $40 \%$ ou mais da área plantada com apenas uma cultura dentro do território de cada município, foi utilizado como critério técnico-econômico para identificar a presença de monocultura e para estabelecer limites e condicionantes à implementação de novos empreendimentos. Nesse sentido, quanto mais alto o índice da monocultura maior deveria ser o grau de restrição para a introdução ou ampliação do eucalipto nos municípios mineiros. Dessa forma, estabeleceu-se, adaptado de Pereira et al. (2008), cinco graus de monocultura mostrados na Tabela 1.

Tabela 1 - Graus de monocultura do eucalipto.

Table 1 - Degrees of monoculture eucalyptus plantations.

\begin{tabular}{lcc}
\hline \multirow{2}{*}{ Graus } & \multicolumn{2}{c}{ Monocultura } \\
\cline { 2 - 3 } & Limite Inferior (\%) & Limite Superior (\%) \\
\hline Muito Baixo & 0 & 10 \\
Baixo & 10 & 20 \\
Médio & 20 & 40 \\
Alto & 40 & 60 \\
Muito Alto & 60 & 100 \\
\hline
\end{tabular}

Fonte: Pereira et al. (2008)

$\mathrm{Na}$ construção desse índice, por meio de dados relativos à área plantada total e à área destinada à plantação de culturas temporárias e permanentes, procurou-se identificar as áreas ocupadas com tais culturas dentro das áreas disponíveis para a atividade agropecuária do municípioe, ainda, as possibilidades de incremento da área agrícola.

\section{RESULTADOS E DISCUSSÃO}

A partir da metodologia descrita, elaborou-se, com base no conjunto de indicadores utilizados no Zoneamento Ecológico-Econômico de Minas Gerais - (ZEE-MG), oÍndice de Monocultura para as plantações florestais, espécie eucalyptus.

Na Tabela 2, observam-se os resultados da aplicação do método proposto para o conjunto dos 10 municípios selecionados. No grupo 1, onde se concentram os municípios com as 5 maiores áreas cultivadas de eucalipto, o grau de monocultura encontrado variou de baixo a médio, com exceção de Antonio Dias, cuja área do território municipal é pequena. Isso significa que nos demais municípios, dentro do critério proposto de limitar a monocultura em até $40 \%$ da área plantada, ainda há espaço para a ampliação das plantações de eucalipto. Naqueles municípios do grupo 2 , onde se observou grau alto e muito alto de monocultura, há restrições de área para a ampliação

Cerne, Lavras, v. 19, n. 1, p. 1-7, jan./mar. 2013 


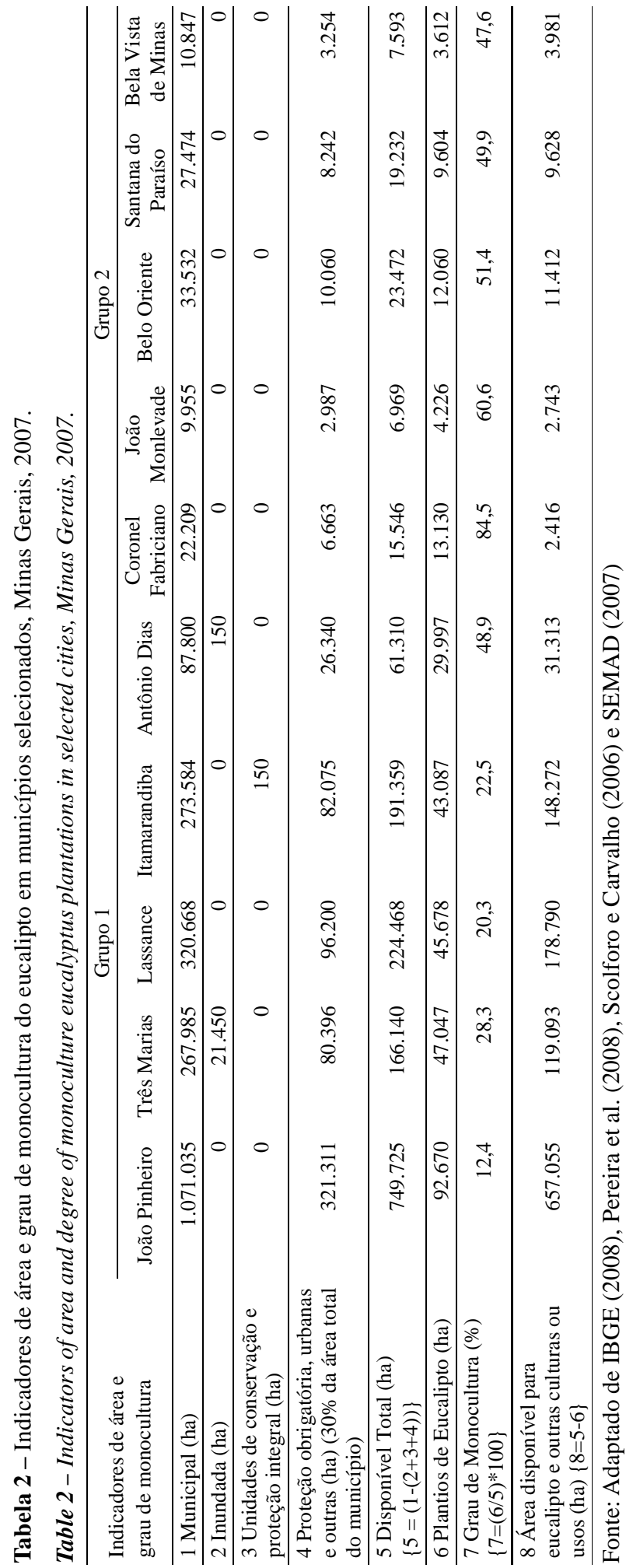

das plantações de eucalipto. Dessa forma, o grau de monocultura pode ser mais um importante indicador de gestão do território dos municípios e do estado de Minas Gerais em relação ao desenvolvimento sustentável da cultura do eucalipto, podendo ser utilizado para outras culturas agrícolas.

Além dos municípios selecionados, a metodologia foi aplicada para o restante dos municípios do estado e os resultados podem ser observados na Figura 1. Em Minas Gerais, existem áreas, em vários municípios e regiões, suficientes para a expansão da silvicultura, com diversidade de clima, solos e vegetação. O índice estadual de monocultura, considerando os estabelecimentos rurais da totalidade dos municípios, alcançou apenas 3,1\%, implicando em muito baixo grau de monocultura.

Há, dessa maneira, possibilidade de expansão da cultura sem concorrer com a produção de alimentos. Tomando-se como referência a área de pastagens naturais e pastagens degradadas, a área potencial de expansão pode ser estimada em cerca de 7 milhões de hectares, ou seja, uma terça parte do total existente informado pelo IBGE (2008). Em algumas regiões, alerta-se que não há mais área agricultável inexplorada a ser incorporada e o crescimento de algumas delas será sempre por substituição de outras culturas. Essa possível área de expansão terá que considerar as condições de solo e clima diversificados, o que torna necessário analisar a aptidão do solo e a disponibilidade de água para possível expansão das áreas de plantações florestais.

A área total do território mineiro é 58,7 milhões de hectares, englobando seus 853 municípios. Os estabelecimentos agropecuários ocupam, de acordo com o Censo Agropecuário (IBGE, 2008), 40,8 milhões de hectares. Considerando que entre 5 e 10\% desse total são formados por áreas degradadas, abandonadas ou impróprias para cultivos agrícolas anuais, tais áreas poderiam ser utilizadas com plantios de espécies florestais de rápido crescimento. Estima-se, portanto, uma área disponível entre 2 e 4 milhões de hectares para a expansão florestal. A realização do plantio florestal nessas áreas possibilitaria a geração de emprego, renda e tributos, além de reduzir a pressão sobre a vegetação nativa e, ainda, recuperar e conservar as áreas degradadas. A expansão das plantações florestais poderia, em sua maioria, ocorrer em forma dos sistemas agroflorestais (ROMANO, 2010), o que contribuiria para recompor a cobertura vegetal, recuperar os solos e fornecer alimentos na forma de grãos, leite e carnes, sem a necessidade de abertura de novas

Cerne, Lavras, v. 19, n. 1, p. 1-7, jan./mar. 2013 


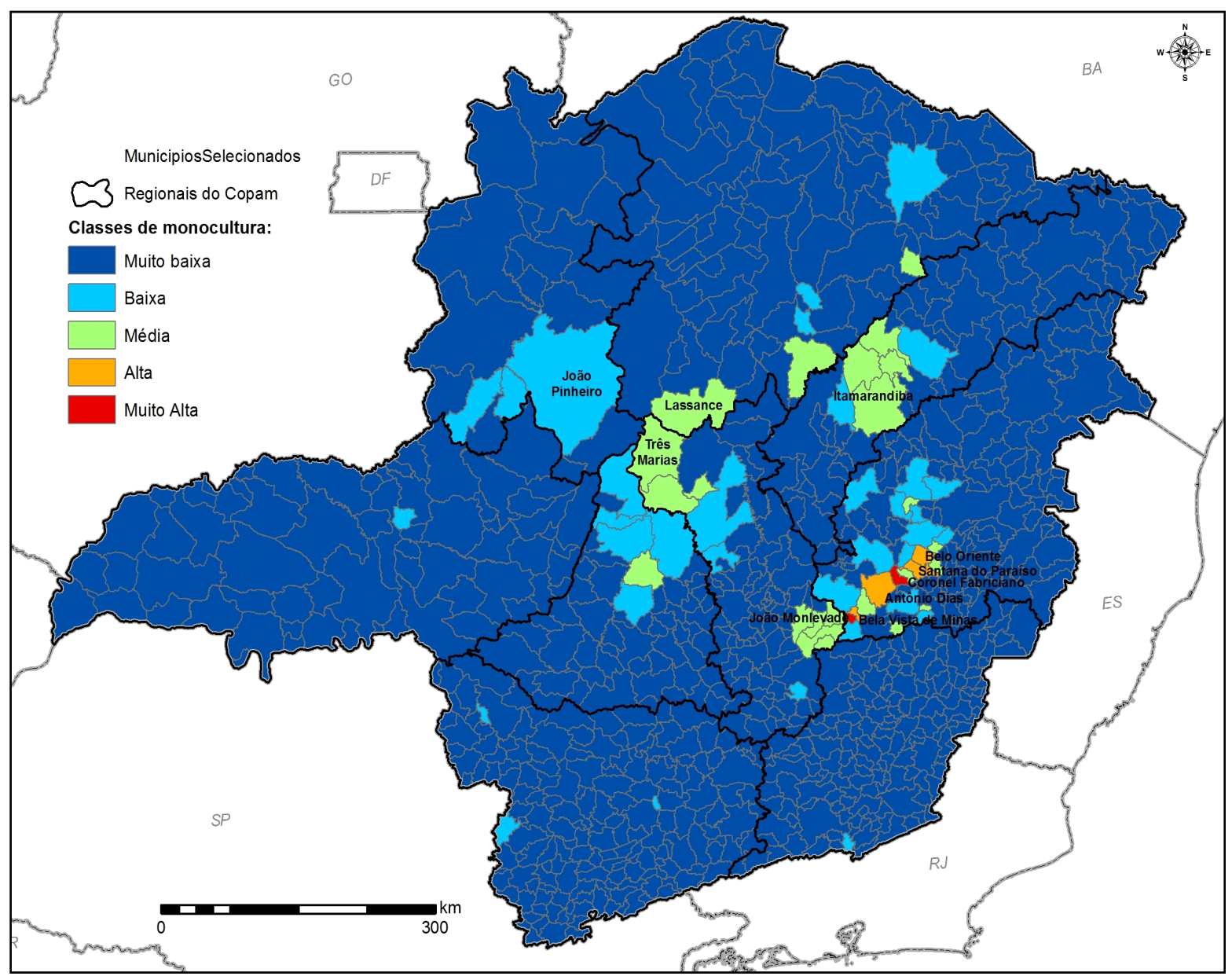

Figura 1 - Índice de Monocultura do Eucalipto para os municípios do estado de Minas Gerais, 2007.

Figure 1 - Index of monoculture eucalyptus plantations for the cities of Minas Gerais, 2007.

áreas, bastando apenas ocupar as áreas atuais de pastagens degradadas. Esses aspectos dizem respeito à gestão do território, entendido aqui no mesmo sentido atribuído por alguns autores (BEDUSCHI FILHO; ABRAMOVAY, 2003; VEIGA, 2001; VIDAL, 2004), ou seja, a gestão territorial, além de consistir na tomada de decisões a respeito da redistribuição de recursos e riquezas já criadas e existentes, deve ressaltar os potenciais para a criação de riquezas, iniciativas e novas formas de coordenação. A abordagem territorial compreende o desenvolvimento além dos limites naturais dos recursos, que passaram a ser conjugados com as condições sociais, econômicas e políticas do território, comoé o caso da expansão da cultura do eucalipto em Minas Gerais. Nesse sentido, Petter et al. (2007) e Wesz Junior (2005) consideram a necessidade de se reconhecer a diversidade dos problemas e das soluções locais para a prática de gestão do território, destacando-se a conjugação de esforços simultâneos nos seguintes setores: o econômico (competitividade territorial); o sociocultural (equidade e diversidade); o ambiental (gestão dos recursos naturais); o político e institucional (democracia e cidadania).

Analisando o resultado para o estado, com base em dados de 2007, como um todo, é possível inferir que a cultura do eucalipto representa um baixo índice de monocultura, com possibilidade de expansão. Na Figura 1, pode-se visualizar os resultados do IM do Eucalipto para todos os municípios do estado de Minas Gerais, onde os municípios destacados em vermelho apresentam os maiores índices de monocultura. A maioria dos municípios não 
atingiu os $40 \%$ de sua área plantada com eucalipto, apresentando muito baixo grau de monocultura à implantação dessa atividade agrícola, expressa pela cor azul na figura.

Com base na metodologia proposta, em Minas Gerais existem áreas, em várias regiões, suficientes para a expansão da silvicultura, com diversidade de clima, solos e vegetação. Dessa forma, deve-se reconhecer as especificidades de cada território, por meio do ZEE-MG, e ofertar instrumentos de desenvolvimento, como o Índice de Monocultura desenvolvido neste trabalho, que atendam às características dos municípios, regiões, estados ou território nacional, uma vez que os territórios constituem palco de novos investimentos, de atração e manutenção de novos empreendimentos.

\section{CONCLUSÕES}

O Índice de Monocultura elaborado neste trabalho alcançou o objetivo proposto de mostrar que a cultura do eucalipto no território de Minas Gerais pode ser expandida sem, no entanto, se caracterizar como monocultura. Dessa forma, esse Índice constitui um instrumento relevante de gestão do território, pois permite planejar, controlar e avaliar a possível expansão do eucalipto, de tal modo a não competir com a produção de alimentos. No âmbito municipal, o Índice permite analisar a geografia dessa cultura e levantar o quantitativo de área disponível para a sua expansão dentro dos limites considerados como não monocultura. Portanto, conclui-se que os resultados apresentados contribuem para a definição de políticas públicas de gestão do território em Minas Gerais, com possibilidades de aplicar a metodologia em outros estados da federação.

\section{REFERÊNCIAS}

ASSOCIAÇÃO BRASILEIRA DE PRODUTORES DE FLORESTAS PLANTADAS. Anuário estatístico da

ABRAF: ano base 2010. Brasília, 2011.

ASSOCIAÇÃO MINEIRA DE SILVICULTURA. Números e índices de Minas Gerais até 2010. Belo Horizonte, 2011. Disponível em: <http://www.silviminas.com.br/usuario/login. aspx?ReturnUrl=/restrito/numerossetor .aspx\%3fano\%3d15\&ano=15>. Acesso em: 16 jun. 2011.

BEDUSCHI FILHO, L. C.; ABRAMOVAY, R. Desafios para a gestão territorial do desenvolvimento sustentável no Brasil. In: CONGRESSO BRASILEIRO DE ECONOMIA E
SOCIOLOGIA RURAL, 41., 2003, Juiz de Fora. Anais... Juiz de Fora: SOBER, 2003. 1 CD-ROM.

INSTITUTO BRASILEIRO DE GEOGRAFIA E ESTATÍSTICA. Censo agropecuário 2006. Rio de Janeiro, 2008. 1 CD-ROM.

KAGEYAMA, A. A. Desenvolvimento rural: conceito e um exemplo de medida. In: CONGRESSO DA SOCIEDADE BRASILEIRA DE ECONOMIA E SOCIALOGIA RURAL, 43., 2004, Cuiabá. Anais... Cuiabá: SOBER, 2004. 1 CDROM.

MINAS GERAIS. Lei $\mathbf{n}^{\mathbf{0}} \mathbf{1 8 . 3 6 5}$, de 01 de setembro de 2009 . Altera a Lei $\mathrm{n}^{\circ} 14.309$, de 19 de junho de 2002, que dispõe sobre as políticas florestal e de proteção à biodiversidade no Estado, e o art. $7^{\circ}$ da Lei Delegada $n^{\circ} 125$, de 25 de janeiro de 2007, que dispõe sobre a estrutura orgânica básica da Secretaria de Estado de Meio Ambiente e Desenvolvimento Sustentável SEMAD. Belo Horizonte, 2009. Disponível em: <http:// www.siam.mg.gov.br/sla/download.pdf?idNorma=10490>. Acesso em: 16 jun. 2011.

NAVARRO, R. M.; HENRÍQUEZ, N. C.; CORNEJO, J. A. The economic and social context or monoculture tree plantations in Chile: the case of the commune or Lumaco, Araucania region. Montevidéo: World Rainforest Movement, 2005. Disponível em: <http:// www.wrm.org.uy/countries/Chile/booklumaco.pdf >. Acesso em: 15 jun. 2011.

PEREIRA, J. R.; REZENDE, J. B.; SILVA, S. S. S.; BOTELHO, D. O.; PEREIRA, N. C. Zoneamento da canade-açúcar e do eucalipto: condicionantes socioeconômicos e índice de monocultura. In: SCOLFORO, J. R.; OLIVEIRA, A. D.; CARVALHO, L. M. T. (Ed.). Zoneamento e cenários exploratórios. Lavras: UFLA, 2008. p. 61-71. Disponível em: 〈http://www.zee.mg.gov.br〉. Acesso em: 20 jan. 2011.

PETTER, R. L.; PETTER, V. L.; QUADROS, F. A. S. Desenvolvimento territorial: uma análise espacial e sua relação com a ruralidade. Revista Brasileira de Agroecologia, Brasília, v. 2, n. 1, p. 938-941, fev. 2007.

ROMANO, P. A. Integração lavoura-pecuária-floresta: uma estratégia para a sustentabilidade. Informe Agropecuário, Belo Horizonte, v. 31, n. 257, p. 7-15, jul./ago. 2010.

Cerne, Lavras, v. 19, n. 1, p. 1-7, jan./mar. 2013 
SCOLFORO, J. R.; CARVALHO, L. M. T. (Ed.).

Mapeamento e inventário da flora nativa e dos reflorestamentos de Minas Gerais. Lavras: UFLA, 2006.

SCOLFORO, J. R. S.; OLIVEIRA, A. D.; CARVALHO, L. M. T. (Ed.). Zoneamento ecológico-econômico do Estado de Minas

Gerais: componente socioeconômico. Lavras: UFLA, 2008. 195 p.

SECRETARIA DE ESTADO DO MEIO AMBIENTE E Desenvolvimento Sustentável. Portal meio ambiente em MG. Disponível em: <http://

www.semad.mg.gov.br/index. php?option = com_content \&task=view\&id=12\&Itemid=26> . Acesso em: 29 nov. 2007.

SPEDDING, C. Sustainable agriculture in developed and developing countries in the future. Réduit: AMAS/Food and Agricultural Research Council, 1997.
VEIGA, J. E. Desenvolvimento territorial do Brasil: do entulho varguista ao zoneamento ecológico-econômico. Bahia Análise \& Dados, Salvador, v. 10, n. 4, p. 193-206, mar. 2001.

VIDAL, S. M. S. C. Gestão território em Santos, SP, e sua interface portuária: apontamentos visando à definição de uma nova relação. In: ENCONTRO NACIONAL PÓSGRAUDAÇÃO EM ADMINISTRAÇÃO, 28., 2004, Curitiba. Anais... Curitiba: ENANPAD, 2004. 1 CDROM.

WESZ JUNIOR, V. J. Desenvolvimento territorial com agroindústrias familiares. In: CONGRESSO BRASILEIRO DE ECONOMIA E SOCIOLOGIA RURAL, 43., 2005, Ribeirão Preto. Anais... Ribeirão Preto: SOBER, 2005. 1 CDROM. 
